\title{
Construction of a Power Quality Monitoring System Based on the Wavelet Transform Change-Point Detection Algorithm on the Virtual Instrument Platform
}

\author{
Shi Lei, Wang $\mathrm{Na}$ \\ Henan Polytechnic Institute, HenanNanyang 473000, China \\ *Corresponding Author Email: shilei8271@163.com
}

Received: April 16, 2020. Revised: October 22, 2020. Accepted: October 23, 2020. Published: October 26, 2020.

\begin{abstract}
It is aimed to carry out the investigation on power quality detection, promote the realization of efficient transmission of network data, and expand the application of wavelet transform change-point detection algorithm in the monitoring system. The voltage deviation is used as a starting point to explore the detection of power quality. First, it describes the harmonics of the public power grid and the limits of harmonic voltage. Second, based on the virtual instrument platform, the power quality monitoring system based on wavelet transform change-point detection algorithm is completed. Finally, by adding a monitoring terminal and a service terminal, the design of the monitoring system server is completed. Through the analysis of the experimental results, it is found that in the monitoring system, the current waveform and the three-phase voltage can be accurately displayed. The combined design of the networked monitoring system and the system server enables the system to complete the rapid transmission of data related to power quality, while having a good monitoring effect. For the optimization of networked monitoring experienceof the server, the application of wavelet transform in power quality measurement is realized. The power quality monitoring system proposed has a strong practicality in power quality monitoring.
\end{abstract}

Keywords - power quality monitoring system, wavelet transform, virtual instrument platform

\section{INTRODUCTION}

Regarding the power quality monitoring, compared with foreign countries, China's works in this field started relatively late. Related electrical energy detection equipment is mostly used for the determination of steady-state parameters, but the accuracy of the measured data is not high enough [1]. The digital signal processing technology and computer information technology developed later make it possible to automate and intelligentize power quality monitoring [2-4]. TAB-Universal series system, Powerlogic power distribution monitoring system and SPM power management system are more commonly used in the monitoring of power quality [5-9]. The application of electric energy is an important indicator of how a country is developing. Although high-power equipment are widely used in China, the problem of electric energy is also prominent [10]. Some of the power equipment in China have poor resistance against electromagnetic interferences, which may not only lead to widespread outages and damage users' economic interests, but also have negative impact on social and economic development [11]. The existing power quality monitoring system has the problems of low acquisition accuracy, single detection index, and lack of online real-time monitoring. In the process of network monitoring, high-precision data acquisition and real-time accurate network transmission are key technical issues.

Based on this, under the composition index related to power quality, the wavelet transform change-point detection algorithm is introduced. The virtual instrument platform is applied to complete the monitoring system for power quality. Furthermore, using virtual instrument technology and wavelet transform change-point detection algorithm, the realization of network monitoring system including monitoring platform and server as well as the application of wavelet transform in power quality monitoring are completed. It is aimed to provide some experimental support and reference for improving the stability and efficiency of the power quality monitoring system.

\section{EXPERIMENT PRINCIPLES AND METHODS}

\section{Voltage Deviation}

In the National Standard Application Manual of Voltage, Current, Frequency and Power Quality, power quality is defined by the National Technical Committee on Voltage, Current Ratings and Frequencies of Standardization Administration of China as follows: deviations in voltage, current or frequency that lead to electrical equipment failures or malfunctioning, including frequency deviation, voltage deviation, power supply reliability, waveform distortion, three imbalances and voltage fluctuation and flicker [12-13]. Generally, voltage deviation is 
the difference between the actual voltage and the system nominal voltage when the variation rate of the power system is less than $1 \%$ per second, represented by the virtual value, as shown in the following formula:

Voltage deviation $(\%)=$ Actual voltage - rated voltage $* 100 \%$

Rated voltage

According to the circuit theory, the effective value of the voltage is:

$U=\sqrt{\frac{1}{T}} \int_{0}^{T} u^{2}(t) d t$

where, $\mathrm{T}$ is the period of the measured signal, and $\mathrm{u}(\mathrm{O}$ is the instantaneous value of the measured voltage. The calculation method for the virtual value can be derived from the definition.

\section{Harmonics in Public Supply Network}

The internationally accepted harmonic wave is defined as: "a sine wave component of a periodic electrical quantity whose frequency is an integral multiple of the fundamental frequency." Regarding harmonics, the following needs to clearly understood:

(1) The number of harmonics must be an integer multiple of the fundamental frequency. For example, the power grid frequency is $50 \mathrm{~Hz}$ in China, so the fundamental frequency is $50 \mathrm{~Hz}$, the second harmonic is $100 \mathrm{~Hz}$ and the third harmonic is $150 \mathrm{~Hz}$ and so on;

(2) Inter-harmonics and sub-harmonics. In the actual power grid, some loads may experience periodically current fluctuations that are not multiples of the fundamental frequency, which is called inter-harmonics; inter-harmonics with a frequency below the fundamental frequency are called sub-harmonics;

(3) Harmonics and transients. Transients and harmonics are two completely different phenomena. Harmonics occur at steady-state conditions and their frequency is an integral multiple of the fundamental frequency. The distorted waveform is continuous or lasts for at least a few seconds. Transient, on the other hand, is a sudden disturbance to the power system. The high frequency of the transient waveform has nothing to do with the fundamental frequency and usually disappears after a few periods.

(4) Short-duration harmonics. Short-duration impulse current can be decomposed according to the periodic function. Inter-harmonics and short-duration harmonics are called fast changing harmonic current or short-time harmonic current, different from steady harmonics and unsteady harmonics;

(5) Trap. When the converter commutates, the voltage waveform will show periodic commutation notches that do not fall within the category of harmonics, which are called traps.

The total harmonic distortion rate (THD) is defined as the ratio between the root-sum-square of the root-mean-square valug of each harmonic component and the root-mean-square value of its fundamental component, as shown in the following formula:

$$
H R U_{k}=\frac{U_{k}}{U_{1}} * 100 \%
$$

\section{Design of the networked monitoring system}

The system uses a distributed network structure, which consists of a power quality monitoring terminal and a monitoring server. As an independent power quality testing terminal, the monitoring terminal collects signals using the NI PCI-6251 board and provides routine power analysis and waveform display; as a node in the distributed network, the client uploads data to the server for monitoring and also uses the server to carry out more complex data analysis and obtains analysis results from it.

The virtual instrument consists of a computer, a hardware interface module and virtual instrument software. The computer is the core part to integrate the hardware interface module and the virtual instrument software. The hardware interface module includes a plug-in DAQ card, a positive EE488 interface (GPIB) card, a VXI controller, and a PXI controller. Through configuration of the test equipment with digital interfaces, the virtual instrument completes such functions as acquisition, amplification and analogue-digital conversion of the measured input signals and digital-to-analogue conversion of output signal (see Figure 1). Therefore, selecting the correct hardware as needed can achieve the optimal system performance. The hardware interface module addresses the signal input and output while the virtual instrument software is the key to the entire system. 


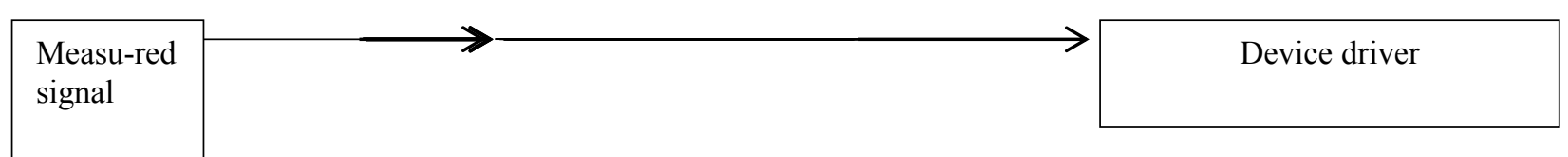

Fig. 1 Virtual instrument structure

To achieve the monitoring of power quality, we must first collect and control the power grid data. The instrument to accomplish this task is called DAQ (data acquisition card). The following indices should be taken into account in the selection of the DAQ card: sampling rate, data resolution and accuracy, input mode, input range, sampling range, gain, noise and isolation performance.

The maximum sampling frequency: the sampling rate determines the rate of $\mathrm{A} / \mathrm{D}$ conversion. The higher the sampling rate is, the more sampling points there will be in a certain period and the more accurate the digital representation of the signals will be. According to the Nyquist Sampling Theorem, the sampling frequency must be greater than twice the maximum frequency of the signal. If multi-channel sampling is implemented, the maximum sampling frequency will be the maximum single-channel frequency divided by the number of channels. Therefore, this parameter needs to be selected based on the frequency of the signal being processed and the number of sampling channels.

\section{Implementation of system servers}

Setting up "Datasocketservermanager": the monitoring terminals IPI (10.13.81.188) and IPZ (10.13.81.181) are added in defaultreaders and defaultwriters so that the monitoring terminals can read and write in the server-side data storage area [14].
Monitoring terminal: the processed voltage deviation rates and frequency deviation rates of the three phases (A, B, and C), three-phase voltage imbalance and three-phase current imbalance are uploaded together to the data buffer area of the server for alarming [15].

Server side: the 8 indices are obtained from the buffer area via Datasocketread and then untied. The upper and lower voltage deviation limits are set up according to the voltage deviation rates and frequency deviation rates of the three phases (A, B, and C) and three-phase imbalances [16].

\section{TEST RESULTS AND ANALYSIS}

\section{Harmonic Voltage}

The limits for harmonic voltage specified in GB/T 14549-93 Quality of Electric Energy Supply - Harmonics in Public Supply Network are listed in Table 1. 
TABLE I Public grid harmonic voltage (phase voltage) limits

\begin{tabular}{|c|c|c|c|}
\hline $\begin{array}{c}\text { Grid } \\
\text { Nominal } \\
\text { Voltage } \\
(\mathrm{kV})\end{array}$ & $\begin{array}{c}\text { Voltage total } \\
\text { harmonic } \\
\text { distortion rate } \\
(\%)\end{array}$ & $\begin{array}{c}\text { Odd } \\
\text { harmonic } \\
\text { voltage } \\
\text { content }(\%)\end{array}$ & $\begin{array}{c}\text { Even harmonic } \\
\text { voltage content } \\
(\%)\end{array}$ \\
\hline 0.38 & 5.0 & 4.0 & 2.0 \\
\hline 6 & 4.0 & 3.2 & 1.6 \\
\hline 10 & 3.0 & 3.2 & 1.6 \\
\hline 35 & 3.0 & 2.4 & 1.2 \\
\hline 66 & 3.0 & 2.4 & 1.2 \\
\hline 110 & 2.0 & 1.6 & 0.8 \\
\hline
\end{tabular}

Even if these equipment are supplied with ideal sinusoidal voltages, they will still generate non-sinusoidal currents, which contain harmonic currents whose content has not much to do with the parameters of the power system but depends heavily on the working conditions, characteristics and working voltages of the equipment. The currents are often seen as the harmonic constant current sources. The harmonics produced by various harmonic sources pollute and harm the power systems mainly in two aspects: first, harm to the electricity, mainly shown in the generation of additional loss and heat to rotating motors or overloaded converter transformers and reduction of equipment life; second, interference to signals: for example, the electromagnetic interferences caused by harmonics will lead to poor communication quality of communication systems. Some sensitive and critical automatic protection and control devices will also make incorrect actions due to harmonics.

The national standard GB 12326-2000 Power Quality - Voltage Fluctuation and Flicker provides the limits, as shown in Table 2 and 3 below. In this standard, the nominal voltage of the system is graded as follows: low voltage (LV) $(\mathrm{U} \mathrm{N} \leq 1 \mathrm{kV})$, medium voltage (MV) $(1 \mathrm{kV}<\mathrm{U} \mathrm{N} \leq 35 \mathrm{kV})$ and high voltage (HV) $(35 \mathrm{kV}<\mathrm{U} N \leq 220 \mathrm{kV})$. The irregular random voltage fluctuations are measured at a probability of $95 \%$. The value marked with "*”" in the table are the limits. 
TABLE II Voltage fluctuation limits

\begin{tabular}{|c|c|c|}
\hline $\begin{array}{c}\text { Frequency of } \\
\text { change } \mathrm{r}(\mathrm{h})\end{array}$ & $\begin{array}{c}\text { LV, MV voltage } \\
\text { fluctuation d (\%) }\end{array}$ & $\begin{array}{c}\text { HV voltage } \\
\text { fluctuation } \mathrm{d}(\%)\end{array}$ \\
\hline $\mathrm{r} \leq 1$ & 4 & 3 \\
\hline $1<\mathrm{r} \leq 10$ & 3 & 2.5 \\
\hline $10<\mathrm{r} \leq 100$ & $2 *$ & 1.5 \\
\hline $100<\mathrm{r} \leq 1000$ & 1.25 & 1 \\
\hline
\end{tabular}

TABLE III Flickers at all levels of voltage limits

\begin{tabular}{|c|c|c|c|}
\hline System voltage level & LV & MV & HV \\
\hline Pst & 1.0 & $0.9(1.0)$ & 0.8 \\
\hline Pit & 0.8 & $0.7(0.8)$ & 0.6 \\
\hline
\end{tabular}

A power supply system has voltage fluctuations because, on one hand, various types of high-power fluctuating loads are put into operation, such as arc furnace, rolling mill, electric locomotive and resistance welding machine, etc.; and on the other hand, the distribution lines are overloaded within a short time and the voltage adjustment ability of power supply interruption is very weak, making it difficult to maintain stable voltage. In addition, household appliances and low-power devices can also cause local voltage fluctuations.

\section{Harmonic Analysis}

In the harmonic analysis of the grid, if FFT transform is used, there will be errors in the analysis results due to the spectrum leakage under nonsynchronous sampling and truncated non-integral period. In addition, since spectrum leakage and noise interference can easily affect the accuracy of the unimodal spectral line correction algorithm, sub-strong spectral lines can be considered in the amplitude correction. Let the fundamental frequency fl be $50 \mathrm{~Hz}$, the sampling frequency fs $12800 \mathrm{~Hz}$, the signal truncation length $\mathrm{N} 2048$ points and the amplitudes (with no unit value) and phases of the fundamental wave and each harmonic are shown in the following table:

TABLE IV Amplitudes and phases of fundamental and harmonics

\begin{tabular}{|c|c|c|c|c|c|c|c|}
\hline Harmonic times & Fundamental wave & 2 & 3 & 4 & 5 & 6 & 7 \\
\hline Magnitude & 220 & 0.5 & 25 & 0.4 & 6 & 0.3 & 3 \\
\hline Phase & $9 \pi / 10$ & $4 \pi / 5$ & $7 \pi / 10$ & $3 \pi / 5$ & $\pi / 2$ & $2 \pi / 5$ & $3 \pi / 10$ \\
\hline
\end{tabular}




\section{Implementation of system}

If the voltage deviation is within the range of the upper and lower limits, it will be displayed as normal; if it is higher than the voltage limit, the alarm will display that the voltage deviation is too high; and if it is lower than the voltage limit, the alarm will display that the voltage deviation is too low [17-18]. The same case is with the frequency deviation. The measurement of voltage imbalance is to take the absolute value of the data, and then compare it with the reference value. If it is greater than the reference value, it indicates that the voltage imbalance exceeds the normal range, and the alarm will display it as abnormal; otherwise, it will display the voltage imbalance as normal [19]. The same case is with the current imbalance. This paper uses the Mallet algorithm to decompose and reconstruct the wavelet and chooses the DB4 wavelet with a filter length of 4 for analysis. The filter coefficients for DB4 wavelet are shown in the following table:

TABLE V Filter coefficients for DB4 wavelet

\begin{tabular}{|c|c|c|c|c|}
\hline $\mathrm{K}$ & 0 & 1 & 2 & 3 \\
\hline $\mathrm{h}(\mathrm{k})$ & $\frac{1+\sqrt{3}}{4 \sqrt{2}}$ & $\frac{3+\sqrt{3}}{4 \sqrt{2}}$ & $\frac{3-\sqrt{3}}{4 \sqrt{2}}$ & $\frac{1-\sqrt{3}}{4 \sqrt{2}}$ \\
\hline $\mathrm{g}(\mathrm{k})$ & $\frac{1-\sqrt{3}}{4 \sqrt{2}}$ & $-\frac{3-\sqrt{3}}{4 \sqrt{2}}$ & $\frac{3+\sqrt{3}}{4 \sqrt{2}}$ & $-\frac{1+\sqrt{3}}{4 \sqrt{2}}$ \\
\hline
\end{tabular}

The principle of the Mallat algorithm for determining the number of decomposition levels is to make the fundamental frequency of the signal located in the centre of the lowest sub-band, thus limiting the impacts of the fundamental frequency component on other sub-bands.

\section{CONCLUSIONS}

This paper programs the networked monitoring system and system server with the aid of the virtual instrument and the wavelet transform change-point detection algorithm. The experiment shows that the current waveform and three-phase voltage can be displayed accurately. The networked monitoring system and system server designed in this experiment can transmit data fast and have good monitoring results, which meets the intended design goals. By giving the system the functions to control user login, set user authorities and record and analyse operational data, this paper further optimizes the networked monitoring experience of the server and fully applies wavelet transform in the measurement of power quality.

The virtual instrument technology is creatively combined with the intelligent detection algorithm. In terms of the amplitude correction of the algorithm, the sub-strong spectral lines are considered, and the Mallet algorithm is used to complete the decomposition and reconstruction of the wavelet. Finally, the monitoring system with efficient data transmission rate and detection accuracy is obtained. However, the application of wavelet transform in power quality measurement is still in the preliminary experimental exploration stage. The further practical application of the power quality system will be the aspect to be further deepened and strengthened in the future.

\section{REFERENCES}

[1] Vidovic, D, Sutlovic, E, Majstrovic, M. "Steady state analysis and modeling of the gas compressor station using the electrical analogy". Energy, 2019, 166(1), 307-317.

[2] Venkatraman, K, Moorthi, S, Selvan, M. P. "Modelling and Control of Transformer-less Universal Power Quality Conditioner (TUnPQC) : An Effective Solution for Power Quality Enhancement in Distribution System". Journal of Control Automation \& Electrical Systems, 2017, 28(1), 123-134. 
[3] Wenge, C, Guo, H, Roehrig, C. "Measurement-based harmonic current modeling of mobile storage for power quality study in the distribution system". Nephron Clinical Practice, 2017, 66(4), 801-814.

[4] Huang W, Dai F. "Research on Digital Protection of Intangible Cultural Heritage Based on Blockchain Technology". Information Management and Computer Science, 2019, 2(1), 14-18.

[5] Chowdhury A H M Y, Hamid MK, Akhi RA. "Impact of Macroeconomic Variables on Economic Growth: Bangladesh Perspective". Information Management and Computer Science, 2019, 2(1), 19-22.

[6] Yakovin, S, Zykov, A, Dudin, S, et al. "Plasma assisted deposition of $\mathrm{TaB}_{2}$ coatings by magnetron sputtering system". Problems of atomic science \& technology, 2017, 107(1), 187-190.

[7] Rim, B. S. “A nonlinear Takagi-Sugeno fuzzy logic control for single machine power system". International Journal of Advanced Manufacturing Technology, 2017, 90(1-4), $1-16$.

[8] Prasad KA. "Impacts Of Agricultural Mechanization: A Case Of Palpa District Of Nepal”. Acta Mechanica Malaysia, 2020, 3(1), 16-19.

[9] Li, Y, Liu, B, Zhang, X, et al. "The distribution of veterinary antibiotics in the river system in a livestock-producing region and interactions between different phases". Environmental Science \& Pollution Research, 2016, 23(16), 16542-16551.

[10] Kumar, R., Singh, B., Shahani, D.T., Jain, C. "Dual-tree complex wavelet transform-based control algorithm for power quality improvement in a distribution system". IEEE Transactions on Industrial Electronics, 2016, 64(1), 764-772.

[11] Garousi, M.R., Shakarami, M.R., Namdari, F. "Detection and classification of power quality disturbances using parallel neural networks based on discrete wavelet transform". Journal of Electrical Systems, 2016, 12(1), 158-173.

[12] Wong V. "Evaluation On Online Learning Resources Based On Customer Satisfaction". Acta Informatica Malaysia, 2020, 4(2), 26-29.
[13] Junior, E.A., Brandao, G.B. "Real time qrs detection based on redundant discrete wavelet transform". IEEE Latin America Transactions, 2016, 14(4), 1662-1668.

[14] Jakovljevic, Z., Puzovic, R., Pajic, M. "Recognition of planar segments in point cloud based on wavelet transform". IEEE Transactions on Industrial Informatics, 2017, 11(2), 342-352.

[15] Zhang, Q., Deng, W. “An adaptive energy management system for electric vehicles based on driving cycle identification and wavelet transform". Energies, 2016, $9(5), 341$.

[16] Jaber, A., Bicker, R. "Industrial robot backlash fault diagnosis based on discrete wavelet transform and artificial neural network". Journal of Quality in Maintenance Engineering, 2016, 11(1), 98-98.

[17] Arslan A. "Computer Network Routing Configuration Based On Intelligent Algorithm. Acta Electronica Malaysia, 2020, 4(1), 28-30.

[18] Yi, H., Xin, S.Y., Yin, J.F. "A class of algorithms for continuous wavelet transform based on the circulant matrix”. Algorithms, 2018, 11(3), 24.

[19] Zhang, H., Fang, J., Liu, H. "Online current signal de-noising of magnetic bearing switching power amplifier based on lifting wavelet transform". Iet Electric Power Applications, 2016, vol.10(8), 799-806.

\section{Creative Commons Attribution License 4.0 (Attribution 4.0 International, CC BY 4.0)}

This article is published under the terms of the Creative Commons Attribution License 4.0 https://creativecommons.org/licenses/by/4.0/deed.en_US 